\title{
Potential biomarkers for alzheimer's disease screened in a small Brazilian population
}

\begin{abstract}
Alzheimer's Disease (AD) leads neurodegenerative diseases ranking and, because of this, clinical attention focus on the identification of blood, serum and plasma' biomarkers in AD patients, since diagnostic have not a gold standard yet. In order to improve diagnose, current study explore relation between biochemical and hematological' parameters, which are commonly applied as lipid profile, liver enzymes, glucose and hemogram. Furthermore, different stages of AD have been evaluated through Clinical Dementia Rating (CDR) and Mini Nutritional Assessment (MNA) evaluated nutritional status, since nutritional status interferes directly in parameters reached. For this purpose, this study included 30 patients, being $18(60 \%)$ women and $12(40 \%)$ men, in different stages of $\mathrm{AD}$. Our data report that malnutrition level is directly related with disease progression, as discussed in literature over the years. With regard to hematological analysis, significate difference was observed between parameters and groups, where patients in moderate stage of disease improved erythrocytes and had greater values of globular volume when compared to final stage, suggesting that $\mathrm{AD}$ is a possible risk factor to anemia. Biochemical data also presented significate difference, showing that disease progression, High Density Lipoprotein (HDL) values and total cholesterol are directly proportional. Take into consideration significate differences from this study and since AD means a heterogeneous disease, more studies are needed to validate in vivo essays that may be relevant to identify this disease.
\end{abstract}

Volume I Issue 4 - 2017

Bárbara Luísa Fermino,' Jéssica Wouk,' Roberta Fabbli, ${ }^{2}$ Weber Claúdio Francisco Nunes da Silva,' José Carlos Rebuglio Vallosa, ${ }^{3}$ Juliana Sartori Bonini,' Luan Henrique Patrzyk, ${ }^{4}$ Anne Karine Bosetto, ${ }^{5}$ Flávia Ivansk ${ }^{5}$

'Pharmacy Department, Midwest State University, Brazil ${ }^{2}$ Department of Neuroscience, University of Florence, Italy ${ }^{3}$ Department of Clinical and Toxicological Analysis, State University of Ponta Grossa, Brazil

${ }^{4}$ Department of Physiotherapy, Midwest State University, Brazil ${ }^{5}$ Department of Biological Sciences, Federal University of Rio Grande do Sul, Brazil

\section{Correspondence: Juliana Sartori Bonini, Pharmacy} Department, Midwest State University, Simeão Camargo Varella de Sá Street, Guarapuava, Paraná, Brazil,

Email Juliana.bonini@gmail.com

Received: September 26, 2017 | Published: October 06, 2017

Keywords: alzheimer disease, biomarkers, neurodegeneration, biochemistry, nutrition

Abbreviations: AD, alzheimer's disease; CDR, clinical dementia rating; MNA, mini nutritional assessment; HDL, high density lipoprotein; LDL, low density lipoprotein levels; $\mathrm{A} \beta$, accumulation of beta-amyloid; NINCDS-ADRDA, national institute of neurologic and communicative disorders and stroke and the alzheimer disease and related disorders association; MMSE, mini-mental state exam; ALT, alanine transaminase; AST, aspartate transaminase; CBC, complete blood count

\section{Introduction}

Alzheimer's disease (AD) is a neurodegenerative disease which presents synaptic loss and neural death, resulting in cerebral atrophy, with consequent and gradual loss of memory and cognitive impairment, individual incapacity and progressive disability. ${ }^{1,2} \mathrm{AD}$ has, as post-mortem markers, insoluble aggregates of $\beta$-amyloid peptides and insoluble filaments composed of hyper phosphorylated tau protein. ${ }^{3}$ To obtain blood samples from patients, such as serum, plasma and circulating cells is simple, therefore these are considered potential sources of biomarkers for AD diagnosis, research and clinical practice., ${ }^{4,5}$ Plasmatic elements such as enzymes, glucose and lipids have been widely studied in $\mathrm{AD}^{\prime}$ 's patients. ${ }^{6}$ Such plasmatic compounds would allow the implementation of effective preventive and therapeutic measures during the early stages of the disease, long before substantial cognitive impairment and brain structure's decline.

Analyses of nutritional blood components and the monitoring of other conditions, have contributed to improve AD's pathological progression understanding. According to Vetrivel and Thinakaran, total cholesterols levels and low density lipoprotein levels (LDL) in serum correlate with the accumulation of Beta-amyloid $(\mathrm{A} \beta)$ peptides in the brain of patients with AD. ${ }^{7}$ Micronutrients, such as selenium and zinc, B vitamins and antioxidant vitamins have being described as fundamental elements for cerebral neuroprotection and cognitive function in elderly people. ${ }^{8}$ According to Chen e Zhong, metabolism dysfunctions of cerebral glucose can be fundamental contributors to the pathogenesis of the disease, through the induction of various factors such as oxidative stress and mitochondrial dysfunction, in addition its occurrence precedes cognitive dysfunction and pathological changes even over the decades. ${ }^{9}$ However, the difficulty of developing a biomarker based in blood for $\mathrm{AD}$ is marked by the fact that Alzheimer's is a disease that progresses slowly and heterogeneously, since mixed pathologies are observed frequently. ${ }^{10}$

Considering such evidences, this study aimed to investigate the relation between biochemical and hematological parameters commonly used in clinical practices, such as lipid profiles, hepatic enzymes, glucose and CBC in patients with Alzheimer's disease, and also correlate them with the different stages of the disease and the nutritional status of the patients.

\section{Materials and methods}

This study was conducted with $\mathrm{AD}$ patients, registered in the "Specialized Component of Pharmaceutical Assistance" of the Health Ministry of Guarapuava, Parana, Brazil, from August to October 2011. Initially, 66 subjects were recruited in the program, with probable diagnosis of $\mathrm{AD}$, according to the criteria of the National Institute of Neurologic and Communicative Disorders and Stroke and the Alzheimer Disease and Related Disorders Association (NINCDS- 
ADRDA). Home visits were made to patients and an Informed Consent Form was provided to the caregivers of the patients. The final sample consisted of 30 patients. This study was approved by the Ethics Committee of the Midwest State University (protocol 026/2011).

For the evaluation of cognitive function, the Mini-Mental State Exam (MMSE) was applied, which enabled the evaluation of spatial and temporal orientation, memory, attention, calculation, language and praxes. The Clinical Dementia Rating (CDR) was utilized to determine the AD stage, according to Morris' criteria, in which a CDR of 0 indicates no dementia and CDRs of $0.5,1,2$ and 3 indicate very mild, mild, moderate, and severe dementia, respectively. ${ }^{11}$ Demographic data of all patients were also collected and included, such as: educational level, age, marital status, average household income, smoking (before the disease), physical activity, comorbidities and medications used.

\section{Nutritional status}

The nutritional status of each subject was assessed through biochemical data, anthropometric data and the MNA (Mini Nutritional Assessment). For biochemical determination of the lipid profile, glycemia, alanine transaminase (ALT) and aspartate transaminase (AST), blood samples were collected after 12 hours of fasting. Samples for the complete blood count (CBC) test were also collected, in order to investigate possible anemia or infection. The blood tests were performed in a private clinical laboratory in the city of Guarapuava.

The anthropometric data includes: height $(\mathrm{m})$, current weight $(\mathrm{kg})$, body mass index (BMI, $\left.\mathrm{kg} / \mathrm{m}^{2}\right)$, mid-upper arm circumference $(\mathrm{MUAC}, \mathrm{cm})$ and calf circumference $(\mathrm{CC}, \mathrm{cm})$. A digital scale with a $150 \mathrm{~kg}$ capacity and measuring tape were used to measure the weight and height respectively. For patients with postural or/and balance difficulty, or bedridden, weight and height were estimated according to average equations proposed by Chumlea. ${ }^{12-14}$

\section{Dietary intake}

Caregivers provided information about the dietary intake of the patients. The dietary intake was assessed based on the 24-hour dietary recall, which lists all the food/beverages, types of food preparations and amounts of each food eaten the day before. The information was collected during a weekday, since the food intake on weekends tends to vary, therefore it cannot be considered a representation of a normal eating pattern for most days. Dietary analysis includes macroand micronutrients values and was performed with the Avanutri 4.0 program (Avanutri Informática LTDA, Brazil).

\section{Statistical analysis}

Descriptive statistics were used, with means and standard deviations for continuous variables and frequency for the categorical ones ANOVA, Kruskal-Wallis or Fisher's exact test were used to compare the three groups of AD stages, in relation to demographic, anthropometric, biochemical, MNA, MMSE and food intake variables. The chi-square test was used to determine the differences between the several methods applied to assess malnutrition and to study possible associations between the usage of some types of medication and nutritional status. Significance was accepted when $\mathrm{P}$ value was $<0.05$. All analyses were performed using SPSS Statistics 19 for Windows (SPSS Inc.).

\section{Results and discussion}

In Table 1 it can be observed that severe dementia (CDR 3) was found in the group with highest average age, being $82.0 \pm 5.4$ years. Additionally, the average age of patients with moderate dementia was $75.0 \pm 8.1$ years and $73.0 \pm 11.5$ years for patients with mild dementia (CDR 1). This data indicates that AD's symptoms worsen with aging. Regarding to gender, in the CDR 1 group 50\% were women and 50\% were men, in the CDR 2 group the prevalence was of women with $62.5 \%$, which also occurred in the CDR 3 group with $66.6 \%$. In terms of physical activity, the most active group was the CDR 1 group and the less active was the CDR 3 group, since $41.6 \%$ of the subjects in this group were bedridden. Among the comorbidities, there was a prevalence of range of blood pressure and several type of depression (which were not classified in the present study).

Table I Features of the patients: age, gender, physical activity and comorbidity in different stages of AD

\begin{tabular}{|c|c|c|c|c|}
\hline & CDR I (n) $n=10$ & CDR $2(n) n=8$ & $\operatorname{CDR} 3(n) n=12$ & $\mathbf{P}^{\mathbf{a}}$ \\
\hline Average age (years) ${ }^{b}$ & $73.0 \pm 11.5^{c}$ & $75.0 \pm 8.1$ & $82.0 \pm 5.4^{c}$ & $0.042^{*}$ \\
\hline Gender & & & & 0,808 \\
\hline Female (\%) & $50.0(5)$ & $62.5(5)$ & $66.6(8)$ & \\
\hline Male (\%) & $50.0(5)$ & $37.5(3)$ & $33.3(4)$ & \\
\hline Physical activity (\%) & & & & $0,028 *$ \\
\hline Bedridden & $0.0(0)$ & $0.0(0)$ & $41.6(5)^{\mathrm{e}}$ & \\
\hline Sedentary & $30.0(3)$ & $50.0(4)$ & $41.6(5)$ & \\
\hline Light Physical Activity & $70.0(7)^{d}$ & $50.0(4)$ & $16.6(2)^{\mathrm{e}}$ & \\
\hline \multicolumn{5}{|l|}{ Comorbidities(\%) } \\
\hline Hypercholesterolemia & $50.0(5)$ & $12.5(I)$ & $33.3(4)$ & 0.303 \\
\hline Diabetes mellitus & $40.0(4)$ & $25.0(2)$ & $33.3(4)$ & 0.889 \\
\hline Parkinson's disease & $0.0(0)$ & $25.0(2)$ & $33.3(4)$ & 0.152 \\
\hline HTN & $60.0(6)$ & $75.0(6)$ & $66.6(8)$ & 0.889 \\
\hline
\end{tabular}


Table Continued....

\begin{tabular}{lllll}
\hline & CDR I (n) $n=10$ & CDR 2 (n) n=8 & CDR 3 (n) n=12 & $P^{\mathbf{a}}$ \\
Depression & $80.0(8)$ & $50.0(4)$ & $58.3(7)$ & 0.398 \\
Stroke & $40.0(4)$ & $25.0(2)$ & $41.6(5)$ & 0.796 \\
Cancer & $20.0(2)$ & $12.5(I)$ & $33.3(4)$ & $0.65 \mid$ \\
\hline
\end{tabular}

AD, alzheimer's disease; HTN, high blood pressure (Hypertension); CDR, clinical dementia rating

$* \mathrm{P}<0.05$ showing a statistically significant difference between groups.

${ }^{a}$ ANOVA or Fisher's Exact test comparing three groups.

${ }^{\mathrm{b}}$ Continuous variables are presented as mean $\pm \mathrm{DP}$.

cMeans with significant differences by the Tukey test $(p<0.05)$.

${ }^{\mathrm{d} C}$ Cells more often observed than the other categories.

${ }^{\mathrm{e} C e l l s ~ l e s s ~ o f t e n ~ o b s e r v e d ~ t h a n ~ t h e ~ o t h e r ~ c a t e g o r i e s . ~}$

Table 2 shows the nutritional status, weight, height, MUAC, CC, BMI, MNA and serum albumin according to the stages of AD. Data showed there is a tendency regarding average weight, height, MUAC,

$\mathrm{CC}$ and $\mathrm{BMI}$, which decreased along the disease progression, but with no significant difference $(p=0,061, p=0,504, p=0,142 ; p=0,081$; $\mathrm{p}=0,101$, respectively).

Table 2 Anthropometric profile, mini nutritional assessment and albumin levels in elderly patients with AD, according to the disease stage

\begin{tabular}{|c|c|c|c|c|}
\hline & CDR I $n=10$ & CDR 2 n=8 & CDR $3 n=12$ & $\mathbf{P a}^{\mathbf{a}}$ \\
\hline Weight (kg) & $\begin{array}{l}70.8 \pm 15.9 \\
(5 ., 2-93.0)\end{array}$ & $\begin{array}{l}61.4 \pm 15.7 \\
(43.6-90.0)\end{array}$ & $\begin{array}{l}56.1 \pm 8.4 \\
(45.5-67.2)\end{array}$ & 0.061 \\
\hline Height (m) & $\begin{array}{l}1.6 \pm 0.10 \\
(1.5-1.8)\end{array}$ & $\begin{array}{l}1.5 \pm 0.05 \\
(1.5-1.6)\end{array}$ & $\begin{array}{l}1.5 \pm 0.1 \\
(1.4-1.7)\end{array}$ & 0.504 \\
\hline MUAC $(\mathrm{cm})$ & $\begin{array}{l}29.0 \pm 5.4 \\
(23.5-39.0)\end{array}$ & $\begin{array}{l}26.0 \pm 4.6 \\
(20.0-33.0)\end{array}$ & $\begin{array}{l}24.9 \pm 2.3 \\
(22.0-29.5)\end{array}$ & 0.142 \\
\hline $\mathrm{CC}(\mathrm{cm})$ & $\begin{array}{l}34.5 \pm 2.9 \\
(30.5-39.5)\end{array}$ & $\begin{array}{l}33.1 \pm 3.6 \\
(26.5-36.5)\end{array}$ & $\begin{array}{l}31.1 \pm 2.7 \\
(27.0-34.5)\end{array}$ & $0.08 I$ \\
\hline BMI $\left(\mathrm{kg} / \mathrm{m}^{2}\right)$ & $\begin{array}{l}26.8 \pm 4.6 \\
(21.8-35.0)\end{array}$ & $\begin{array}{l}24.6 \pm 6.5 \\
(17.1-36.5)\end{array}$ & $\begin{array}{l}22.8 \pm 3.5 \\
(17.0-30)\end{array}$ & 0.101 \\
\hline MNA (score) & $\begin{array}{l}21.9 \pm 2,3^{b} \\
(17.0-26.0)\end{array}$ & $\begin{array}{l}19.3 \pm 4.8 \\
(14.0-26.0)\end{array}$ & $\begin{array}{l}17.2 \pm 4.2^{b} \\
(10.0-22.0)\end{array}$ & $0.042 *$ \\
\hline
\end{tabular}

MUAC, mid-upper arm circumference; $C C$, calf circumference; BMI, body mass index; MNA, mini nutritional assessment

Data is presented as mean \pm DP (amplitude).

${ }^{a}$ Kruskal Wallis Test.

$b, c, d$ Means with significant differences among themselves by Dunn Test $(p<0.05)$.

$* \mathrm{P}<0.05$ showing a statistically significant difference between groups.

Table 3 shows the hematological profile according to the progression of AD. Values of erythrocytes $(p=0,01)$, hemoglobin $(\mathrm{p}=0,06)$ and globular volume $(\mathrm{p}=0,02)$ decreased, comparing the CDR 2 group to the CDR 3 group, however, only the erythrocytes and globular volume were significant. Furthermore, values of leukocytes and neutrophils were within the normal range for all 3 groups, thus excluding acute inflammatory processes.

After analyzing the biochemical profile of elderly AD patients of this study (Table 4), we can notice a decrease tendency in total cholesterol and high density lipoprotein (HDL) cholesterol levels in the CDR 2 group when compared to CDR 3 group. Triglyceride levels

demonstrated a decrease tendency along the disease progression, the same occurred to LDL cholesterol levels between the groups CDR 2 and CDR $3(111,7 \pm 14,4$ in the CDR 2 group to $89,3 \pm 21,1$ in the CDR 3 group), however these differences were not significant.

When comparing this study with another brazilian one performed in the country's south region, by Maia et al., with patients that had different types of dementias, it was observed a difference in the distribution among the stages of the disease, in which $21.4 \%(n=55)$ presented very mild dementia (CRD 0.5), 26.8\% $(n=69)$ were in the mild stage of the disease (CDR 1), 32.6\% $(n=84)$ in the moderate stage (CDR 2) and 19.1\% $(n=49)$ in the severe stage (CDR 3$).{ }^{15}$ The 
frequency was greater among elderly female patients with $66.66 \%$ (8 patients). These data corroborate studies made by Holanda, Ponte and Pinheiro, in which the majority of the subjects were in the most severe stage of the disease, with a predominance of female patients corresponding $40 \%$ of the total. ${ }^{16}$

Table 3 Hematological profile of elderly people with AD, according to the disease stages

\begin{tabular}{|c|c|c|c|c|c|c|}
\hline & CDR I $(n=10)$ & CDR $2(n=8)$ & CDR $3(n=\mid 2)$ & $\mathbf{F}$ & $P$ & Dif \\
\hline Erythro & $4.6 \pm 0.4$ & $5.0 \pm 0.4$ & $4.3 \pm 0.4$ & 5.5 & 0.01 & $2>3$ \\
\hline Hemo & $13.7 \pm 1.6$ & $14.7 \pm 1.2$ & $12.8 \pm 1.4$ & 3.2 & 0.06 & \\
\hline Glob.Vol. & $4 I, 4 \pm 3,3$ & $44,4 \pm 2,7$ & $38,8 \pm 3,8$ & 5,0 & 0,02 & $2>3$ \\
\hline VGM & $81,5 \pm 26,0$ & $89,8 \pm 3,8$ & $91,0 \pm 3,7$ & 0,9 & 0,44 & \\
\hline HGM & $29,4 \pm 2,6$ & $29,6 \pm 0,8$ & $29,9 \pm 1,8$ & 0,2 & 0,85 & \\
\hline CHGM & $32,9 \pm 1,4$ & $33,0 \pm 0,8$ & $32,9 \pm 1,0$ & 0,0 & 0,98 & \\
\hline RDW & $13,6 \pm 0,9$ & $13,0 \pm 0,4$ & $13,3 \pm 0,7$ & $\mathrm{I}, \mathrm{I}$ & 0,35 & \\
\hline Leuco & $7464,0 \pm 2806,5$ & $8206,7 \pm 1828,2$ & $7437,8 \pm 2164,0$ & 0,2 & 0,80 & \\
\hline Eosinophils & $201,6 \pm 166,9$ & $757,4 \pm 87 \mid, 2$ & $244,4 \pm 237,7$ & 3,2 & 0,06 & \\
\hline Linfo & $2292,5 \pm 664,1$ & $1826,1 \pm 1064,2$ & $2065,2 \pm 468,4$ & 0,8 & 0,46 & \\
\hline Monocytes & $572,0 \pm 157,2$ & $610,1 \pm 180,3$ & $698,8 \pm 274,9$ & 0,9 & 0,43 & \\
\hline Rods & $84,6 \pm 35,2$ & $|34,| \pm 8||$, & $94,6 \pm 70,0$ & 1,3 & 0,30 & \\
\hline Segmented & $43 \mid 3,3 \pm 2480,5$ & $4625,4 \pm 1186,9$ & $4324,6 \pm \mid 832,1$ & 0,1 & 0,95 & \\
\hline Neutrophils & $4397,9 \pm 2498,1$ & $4759,4 \pm 1206,2$ & $44 \mid 9,2 \pm 1860,3$ & 0,1 & 0,93 & \\
\hline Platelets & $225500,0 \pm 56 \mid 27,3$ & $247|66,7 \pm 8| 330,0$ & $216666,7 \pm 44886,0$ & 0,5 & 0,62 & \\
\hline
\end{tabular}

Dif, significant comparisons; TE, effect size; Adjusted for covariable age

Table 4 Biochemical profile of elderly people with AD, according to the disease stages

\begin{tabular}{lllllll}
\hline & CDR I $(\mathbf{n = 1 0})$ & CDR 2 $(\mathbf{n = 8})$ & CDR 3 $(\mathbf{n = 1 2})$ & F & $P$ & Dif \\
\hline HDL & $44,3 \pm 8,7$ & $54,0 \pm 11,6$ & $39,3 \pm 7,4$ & 4,8 & 0,02 & $2>3$ \\
LDL & $93,7 \pm 26,0$ & $|1|, 7 \pm 14,4$ & $89,3 \pm 21,1$ & 2,0 & 0,16 & \\
Total Col. & $166,3 \pm 24,9$ & $191,5 \pm 18,6$ & $150,2 \pm 18,0$ & 6,8 & 0,01 & $2>3$ \\
VLDL & $28,0 \pm 13,0$ & $25,8 \pm 15,6$ & $21,4 \pm 7,0$ & 0,7 & 0,49 & \\
Glucose & $92,4 \pm 35,2$ & $89,8 \pm 8,8$ & $86,8 \pm 19,3$ & 0,1 & 0,89 & \\
AST & $32,5 \pm 7,5$ & $25,3 \pm 6,6$ & $24,9 \pm 6,5$ & 3,4 & 0,05 & $1>2$ \\
ALT & $30,4 \pm 20,7$ & $18,3 \pm 6,8$ & $17,8 \pm 6,6$ & 2,3 & 0,12 & \\
Tri & $139,9 \pm 64,7$ & $129,8 \pm 78,8$ & $106,4 \pm 35,3$ & 0,8 & 0,48 & \\
\hline
\end{tabular}

Dif, significant comparisons; TE, effect size; Adjusted for covariable age

The group of patients with AD in severe stage (CDR 3) showed a greater weight loss, however with no significant difference when compared to other groups, and in this group the average age was higher, with $82.0 \pm 5.4$ years. Also, this group had the highest percentage of bedridden elderlies, which can cause the loss of body mass, resulting in weight loss. The agnosia and apraxia, symptoms arising from this dementia, decrease energy intake accelerating the weight loss process, making the patient more and more dependent upon their caregivers. Swallowing disorders such as dysphagia, also affect individuals with $\mathrm{AD}$, affecting food intake along with anorexia caused by the atrophy of the MTL. ${ }^{17,18}$

The MNA showed that in the CDR 3 group, half of the patients demonstrated malnutrition and the other half had the risk of developing this condition. In this context, we emphasize the importance of nutritional monitoring, as well as of ensuring the intake of adequate micronutrients, either with oral supplementation or nutrition education, in order to try to minimize the occurrence of malnutrition in elderlies. ${ }^{19}$

According to Wong et al., ${ }^{20}$ individuals with high serum levels of total cholesterol may have increased risk of developing $\mathrm{AD} .{ }^{20}$ However, Beeri et al. ${ }^{21}$ found no correlation between total cholesterol levels and decline of cognition. ${ }^{21}$ In contrast, according to Van Vliet ${ }^{22}$ low cholesterol levels may impair brain function, since cholesterol is essential for synapses formation, playing an important role in the regulation of signal transduction. ${ }^{22}$ However, the cholesterol membrane also plays a role in the formation and aggregation of $\beta$-amyloid, thanks 
to apolipoprotein $\mathrm{E}$ (apoE). There are two models that aim to explain the role of apoE in $A \beta$ accumulation. In the first one, it is suggested that soluble $\mathrm{A} \beta$ peptides interact with apoE which is associated with a lipid particle, following to an endocytosis mediated by a receptor. Lipoproteins are digested by enzymes in lysosomal compartment, releasing cholesterol to the cell. In lysosomes, a fraction of ApoE-A $\beta$ is degraded, while the rest of it remains associated with $A \beta$, promoting its aggregation to amyloid fibrils, that are secreted back to extracellular mean. ApoE e 4 has more affinity to $A \beta$ than to other isoforms, therefore a more accelerated process is expected. In the second model, it is suggested that beyond facilitating directly the entrance of $A \beta$ in the cell and also helping in its aggregation process, $A p o E$ could increase the rate of $A \beta$ production through the enhancement of cholesterol levels in the cell. After endocytosis mediated by a receptor and enzymatic digestion of lipoproteins, cholesterol is released to cell membranes. Lipoproteins such as ApoE e4 tend to contain more cholesterol, and the increase of sterol in intracellular membranes may promote an enhancement of $\mathrm{A} \beta$ generation rate, resulting in a greater release of it to extracellular mean. ${ }^{23,24}$

According Bortoli et al., ${ }^{25,26}$ decreased HDL values are usually related to lifestyle and diet habits, factors that with the progression of $\mathrm{AD}$ are increasingly compromised. ${ }^{25,26}$ According to Hottman et al., ${ }^{27}$ evidences indicate that HDL modulates the cognitive function in ageing and neurodegenerative diseases associated with ageing, however the underlying mechanisms by which HDL modulates the cognitive function are poorly understood, being often related to apoE genotypes and its isoforms, such as ApoE2, ApoE3 and ApoE4. ${ }^{27}$ When the levels of HDL cholesterol are observed in serum ApoE4, these isoforms are found opposed to LDL cholesterol. ApoE2 is a protector isoform regarding to neurodegenerative diseases, because it is able to generate HDL particles, promoting the maintenance of myelinated neurons. ${ }^{20,28}$ It is important to mention that clinical studies in different ethnic populations have shown that high HDL levels in plasma were associated with a decreased risk for developing $\mathrm{AD}$, possibly due to genetic aspects regarding to ApoE isoforms and their capability to generate different lipoprotein molecules. ${ }^{29}$

There is no scientific evidence that the blood test alone can serve as a peripheral biomarker for Alzheimer's, however, it is commonly used in clinical practice associated with other laboratory blood tests, like concentration of creatinine in serum, TSH, albumin, liver enzymes, vitamin B12, folic acid, calcium, and serological reactions for syphilis, in order to exclude other possible potential secondary causes of dementia. ${ }^{4,5}$

\section{Conclusion}

In this work, it was possible to observe a great discrepancy between the results of analyzed parameters, what hampers the study of $\mathrm{AD}$ blood biomarkers. Blood analyses of each patient must be individually interpreted, considering their comorbidities and medications used that may interfere in the diagnosis, as alterations in blood biomarkers may occur due to these comorbidities or to AD. Regardless of each biomarker is chosen to be studied, there is a need to standardize and validate the technical aspects of the acquisition, measurement and analyzes of samples, from both animals and humans. It is known that in the biochemical field there are variations attributed to non-biological factors such as equipment, and data acquisition and analysis that may be the main sources of error. A single biomarker should not be used in an attempt to guide decision making. Biomarkers are most useful when used as guides together with other complementary data

\section{Acknowledgements}

This work was supported by National Council for Scientific and Technological Development (CNPq), National Council for the Improvement of Higher Education (CAPES), State of Parana Research Foundation and Association of Study, Research and Assistance to patients with Alzheimer's disease (AEPAPA).

\section{Conflict of interest}

The authors declared no potential conflicts of interest with respect to the research, authorship, and/or publication of this article.

\section{References}

1. Weiner MW, Veitch DP, Aisen PS, et al. 2014 update of the alzheimer's disease neuroimaging initiative: A review of papers published since its inception. Alzheimers Dement. 2015;11(6):e1-120.

2. Tousseyn T, Bajsarowicz K, Sánchez H, et al. Prion disease induces alzheimer disease-like neuropathologic changes. J Neuropathol Exp Neurol. 2015;74(9):873-888.

3. Sheng M, Sabatini BL, Sudhof TC. Synapses and alzheimer's disease. Cold Spring Harb Perspect Biol. 2012;4(5):a005777.

4. Caramelli P, Barbosa TM, Sakurai E, et al. The Pietà study: epidemiological investigation on successful brain aging in Caeté (MG), Brazil. Methods and baseline cohort characteristics. Arq Neuro Psiquiatr. 2011;69(4):579-584.

5. Caramelli P, Teixeira LA, Buchpiguel AC, et al. Diagnosis of Alzheimer's disease in Brazil: Supplementary exams. Dement neuropsychol. 2011;5(3):167-177.

6. Patil SP, Ballard R, Sanchez S, et al. ApoE: the link between Alzheimer'srelated glucose hypometabolism and A $\beta$ deposition? Med Hypotheses. 2012;78(4):494-496.

7. Vetrivel KS, Thinakaran G. Membrane rafts in Alzheimer's disease betaamyloid production. Biochimic Biophysica Acta. 2010;1801(8):860-867.

8. Ferry M, Roussel AM. Micronutrient status and cognitive decline in ageing. Eur Geriatr Med. 2011;2(1):15-21.

9. Chen Z, Zhong C. Decoding Alzheimer's disease from perturbed cerebral glucose metabolism: implications for diagnostic and therapeutic strategies. Prog Neurobiol. 2013;108:21-43.

10. Whitwell J, Duffy JR, Strand EA, et al. Clinical and neuroimaging biomarkers of amyloid-negative logopenic primary progressive aphasia. Brain Lang. 2015;142:45-53.

11. Morris JC, Mckeel DW, Storandt M, et al. Very mild Alzheimer's disease Informant-based clinical, psychometric, and pathologic distinction from normal aging. Neurology. 1991;41(4):469-478.

12. Chumlea WC, Roche AF, Steinbaugh ML. Estimating stature from knee height for persons 60 to 90 years of age. J Am Geriatr Soc. 1985;33(2):116-120.

13. Chumlea WC, Baumgartner R, Roche AF. Specific resistivity used to estimate fat-free mass from segmental body measures of bioelectric impedance. Am J Clin Nutr. 1988;48(1):7-15.

14. Chumlea WC, Guo S, Roche AF, et al. Prediction of body weight for the nonambulatory elderly from anthropometry. $J$ Am Diet Assoc. 1988;88(5):564-568.

15. Maia ALG, Godinho C, Ferreira ED, et al. Application of the Brazilian version of the CDR scale in samples of dementia patients. Arq Neuro Psiquiatr. 2006;64(2b):485-448. 
16. Holanda ÍTA, Pinheiro MCD, Ponte KMDA. Idosos com Alzheimer: um estudo descritivo. Rev Rene. 2012;13(3):582-89.

17. Muñoz AM, Agudelo GM, Lopera FJ. Diagnóstico del estado nutriciona de los pacientes con demencia tipo Alzheimer registrados en el Grupo de Neurocienciais, Medellín, 2004. Biomédica. 2006;26(1):113-25.

18. Goes VF, Mello Carpes PB, De Oliveira LO, et al. Evaluation of dysphagia risk, nutritional status and caloric intake in elderly patients with Alzheimer's. Rev Latino Am Enfermagem. 2014;22(2):317-324.

19. Pivi GA, Da Silva RV, Juliano Y, et al. A prospective study of nutrition education and oral nutritional supplementation in patients with Alzheimer's disease. Nutr J. 2011;10:98.

20. Wong MW, Braidy N, Poljak A, et al. Dysregulation of lipids in Alzheimer's disease and their role as potential biomarkers. Alzheimers Dement. 2017;13(7):810-827.

21. Beeri MS, Ravona Springer R, Silverman MJ, et al. The effects of cardiovascular risk factors on cognitive compromise. Dialogues Clin Neurosci. 2009;11(2):201-212.

22. Van Vliet P. Cholesterol and late-life cognitive decline. J Alzheimers Dis. 2012;30(Suppl 2):S147-162.

23. Pohlkamp T, Wasser CR, Herz J. Functional roles of the interaction of app and lipoprotein receptors. Front Mol Neurosci. 2017;10:54.
24. Hu J, Liu CC, Chen XF, et al. Opposing effects of viral mediated brain expression of apolipoprotein E2 (apoE2) and apoE4 on apoE lipidation and $\mathrm{A} \beta$ metabolism in apoE4-targeted replacement mice. Molecular Neurodegeneration. 2015;10:6.

25. Bortoli $\mathrm{C}$, Bonatto $\mathrm{S}$, Bruscato $\mathrm{MN}$, et al. Intake of saturated fat and carbohydrates among adult and elderly patients with dyslipidemia in the Veranópolis project. Rev Bras Cardiol. 2011;24(1):33-41.

26. Dias IH, Polidori MC, Li L, et al. Plasma levels of HDL and carotenoids are lower in dementia patients with vascular comorbidities. J Alzheimers Dis. 2014;40(2):399-408.

27. Hottman DA, Chernick D, Cheng S, et al. HDL and cognition in neurodegenerative disorders. Neurobiol Dis. 2014;72(pt A):22-36.

28. Conejero Goldberg C, Gomar JJ, Bobes Bascaran T, et al. APOE2 enhances neuroprotection against Alzheimer's disease through multiple molecular mechanisms. Mol Psychiatry. 2014;19(11):1243-1250.

29. Reitz C, Tang MX, Schupf N, et al. Association of higher levels of highdensity lipoprotein cholesterol in elderly individuals and lower risk of late-onset alzheimer disease. Archives of Neurology. 2010;67(12):14910 1497. 